\section{Craniofacial Morphology Affects Bite Force in Patients with Painful Temporomandibular Disorders}

Paula Furlan Bavia, Larissa Soares Reis Vilanova, Renata Cunha Matheus Rodrigues Garcia
Department of Prosthodontics and Periodontology, Piracicaba Dental School, UNICAMP

- Universidade Estadual de

Campinas, Piracicaba, SP, Brazil

Correspondence: Renata Cunha Matheus Rodrigues Garcia, Av. Limeira, $n^{\circ}$ 901, Bairro Areião, 13414-903 Piracicaba, SP, Brasil. Tel: +55-19-2106-5240. e-mail: regarcia@fop.unicamp.br
Craniofacial morphology affects masticatory performance in healthy dentate subjects, but little is known about its effects in patients with painful temporomandibular disorders (TMDs). Forty-eight female patients (mean age of $28 \pm 5.8$ years) with painful TMDs underwent lateral cephalometric radiography. Using Ricketts' cephalometric analysis and the Vert method, subjects were assigned to three groups according to their craniofacial morphology: brachyfacial $(n=22)$, mesofacial $(n=13)$, and dolichofacial $(n=13)$. Research diagnostic criteria for TMD were used to confirm the TMD diagnosis for each patient. Pain intensity was reported by each patient based on a visual analog scale (VAS). Maximum bite force (MBF) was measured with pressure sensors placed on the first molar site. Masticatory performance (MP) was assessed by chewing a silicone-based artificial material and determining the resulting particle size by the sieve method. Chewing ability (CA) was evaluated for seven food types and analyzed by a VAS questionnaire. Data were analyzed by one-way ANOVA followed by a Tukey-Kramer test $(p<0.05)$. MBF differed in each group, with brachyfacial patients having the highest MBF values. There was no difference in MP among the groups. The groups differed only in their ability to chew one of the seven evaluated food types. In summary, craniofacial morphology affects the MBF without impairing MP or CA in patients with painful TMDs.
Key Words: temporomandibular disorder, craniofacial morphology, bite force, mastication.

\section{Introduction}

Mastication is the first step in breaking down food into smaller particles to facilitate enzymatic digestion (1). The central nervous system coordinates this function of the stomatognathic system and it may be altered by several factors, such as dental occlusion, neuromuscular dysfunction, periodontal mechanoreceptors and pain (2). Thus, functional alterations of the stomatognathic system may result in poor masticatory function, which could lead to poor nutrition and, ultimately, poor health (3).

Bite force (BF) is an important component in masticatory function assessment. BF is responsible for the process of comminuting food in preparation for swallowing. Several factors, such as the number of occlusal pairs (4), masticatory muscle thickness (4), and malocclusion (5), can negatively influence BF and, consequently, impair masticatory function. BF can be affected by craniofacial morphology, as it has also been demonstrated. Dolichofacial patients have lower BF values than patients with other craniofacial morphologies $(6,7)$. Temporomandibular disorders (TMDs), such as disc displacement with reduction (8) and temporomandibular joint (TMJ) or muscle pain (6), can also decrease $B F$, suggesting poorer masticatory function in such subjects.

Masticatory function can also be assessed by masticatory performance (MP) and chewing ability $(C A)$ tests $(9,10)$. The first measures the particle size of chewable test materials after a given number of chewing strokes $(9,10)$, whereas the latter involves the subjects' self-perception in the evaluation of their masticatory function (10). MP is reduced in pain-free dolichofacial subjects, regardless if they are dentate or edentulous patients fitted with implantsupported overdentures or conventional mandibular dentures $(7,11)$. However, the relationship between CA and types of facial morphology in asymptomatic subjects is unknown. In subjects with TMD symptoms, studies have shown decreased MP and CA values $(1,12)$. According to some authors, this reduced masticatory function in TMD patients may be due to pain during chewing, which causes these patients to perform slower and smaller movements in an attempt to avoid aggravation of the injury (13).

The literature shows few reports on the relationship between BF, TMD and craniofacial morphology (14-16) with methodological and population differences; however, no studies were found to investigate the influence of the craniofacial morphology on MP and CA in subjects with painful TMDs. The hypothesis is that the craniofacial morphology may alter the mastication function in individuals with painful TMD. Thus, the aim of this study was to evaluate the maximum BF (MBF), MP and CA in painful TMD subjects, considering different craniofacial 
morphologies.

\section{Material and Methods}

Subjects

Forty-eight female subjects with painful TMDs, aged $18-45$ years (mean: $28 \pm 5.8$ years), participated in this study. These subjects were selected from a cohort of patients who sought care for facial pain as well as volunteer TMD patients from the student body and staff of Piracicaba Dental School, University of Campinas, Brazil. To be included in the study, patients were required to have complete dentition, good oral and general health, and experienced pain in the masticatory muscles and/or TMJ for at least 3 months.

Pain intensity was assessed by using a visual analog scale (VAS). The VAS was a 100-mm-long horizontal line, anchored by word descriptors at each end. The left side of the scale $(0 \mathrm{~mm})$ read "no pain," and the right side of the scale $(100 \mathrm{~mm})$ read "worst pain imaginable". Each subject drew a vertical mark on the line at the point that best represented the level of their perceived pain. Only subjects presenting a pain intensity of $50 \mathrm{~mm}$ or more on the VAS, for at least 3 months were selected. The VAS was applied during the subject's recruitment and confirmed during TMD diagnosis session. Individuals presenting severe malocclusion (e.g., anterior open bite, deep bite, unilateral or bilateral posterior crossbite), previous maxillofacial surgery, current treatment for neurological or psychological disorders, parafunctional habits, facial deformities, pregnancy, removable partial or complete dentures and/or intraoral appliances, and those receiving TMD treatment or drug therapies were excluded from the study.

All participants underwent conventional lateral cephalograms followed by Ricketts' cephalometric analysis to determine their craniofacial morphology. Based on the results of this analysis, subjects were classified as brachyfacial $(n=22)$, mesofacial $(n=13)$, or dolichofacial $(n=13)$. All subjects signed a consent form approved by the Ethics Committee of Piracicaba Dental School, University of Campinas (protocol \# 022/2012). The study was registered in the Brazilian Registry of Clinical Trials (No. RBR-87rdwv).

Masticatory function was objectively assessed by $\mathrm{MBF}$ and MP assessments and a subjective test for CA was administered. All evaluations, including the TMD diagnosis, were performed by a single researcher, blinded to each participant's assigned craniofacial morphology classification.

All participants also received an anthropometric evaluation. Body height (in $\mathrm{m}$ ) was measured with the subject in the upright position. Weight (in $\mathrm{kg}$ ) was measured with a mechanical scale (MIC 1/C A; Micheletti, São Paulo, SP, Brazil). Body mass index (BMI) was calculated as the subject's weight divided by the square of their height $\left(\mathrm{kg} / \mathrm{m}^{2}\right)$.

\section{TMD Diagnosis}

All participants were subjected to clinical examination for diagnosis of TMD by application of the Research Diagnostic Criteria for TMD (RDC/TMD) Axis I protocol. A single researcher performed the RDC/TMD examination of all subjects. This researcher was previously calibrated by two examiners, one of whom is an expert RDC/TMD examiner. The Kappa index was 0.96 (95\% confidence interval, 0.89-1.00), confirming that the study researcher could accurately diagnose patients using the RDC/TMD protocol. The included subjects fell into one or more RDC/ TMD diagnostic groups. RDC Group I patients presented myofascial pain with or without limited mouth opening. RDC Group II patients presented disc displacement with or without reduction and with or without a limited mouth opening. RDC Group III patients presented arthralgia, osteoarthritis or osteoarthrosis.

\section{Craniofacial Morphology}

Lateral cephalograms were used to determine craniofacial morphology. During each radiographic procedure, the subject was protected by a lead apron. After position in the cephalostat, the subject's teeth were held in the maximal intercuspal position and the lips were lightly closed. The subject's sagittal plane was perpendicular to the path of the $x$-ray. Their Frankfort (horizontal) plane was parallel to the floor (17). All cephalograms were obtained with the same radiographic unit (Kodak $8000 \mathrm{C}$, Eastman Kodak Company, France), using standard procedures. A single investigator, blinded to the RDC/TMD diagnosis of the patient, performed all cephalometric analyses using Radiocef Studio 2.0 software (Radiomemory, Belo Horizonte, MG, Brazil).

The vertical facial pattern was determined by calculating the VERT Index suggested by Ricketts et al. (18), using the following five mandibular measurements: facial axis length, anterior lower facial height, mandibular plane length, mandibular arch length and facial depth. The Vert Index is the arithmetic mean of the difference between each of these five cephalometric measures and the value considered ideal for a harmonic face, divided by the standard deviation (19). Each subject was classified as dolichofacial (Vert index below -0.5$)$, mesofacial (Vert index between -0.49 and +0.49 ), and brachyfacial (Vert index above +0.5$)(18)$. The Vert index is negative when the growth trend is vertical and positive when the growth trend is horizontal.

\section{$M B F$}

MBF was measured with a BF transducer (Spider 8; Hottinger Baldwin Messtechnik $\mathrm{GmbH}$, Darmstadt, 
Germany) (20). BF sensors had a diameter of $12.7 \mathrm{~mm}$ and were $0.25 \mathrm{~mm}$ thick (FSR no. 151; Interlink Electronics Inc., Camarillo, CA, USA). Sensors were protected from deformities during clenching on both sides by $0.7-\mathrm{mm}$-thick metal disks (7) and $1.7 \mathrm{~mm}$ wide rubber disks, resulting in a 5.05-mm-wide assembly. The assemblies were protected from humidity with a plastic film and were positioned in the bilateral first molar site. Subjects were instructed to occlude with maximum force for $7 \mathrm{~s}$ (7). The procedure was repeated after $5 \mathrm{~min}$, and the average of the two measurements was used as the MBF value, expressed as kilogram-force (kgf). During the procedure, the applied force was amplified, recorded and analyzed with Catman Easy software (ver. 1.0, Hottinger Baldwin).

\section{MP}

MP was evaluated by the sieve method. Subjects were asked to chew 17 cubes of an artificial silicon test material (Optosil; Hereaus Kulzer, São Paulo, SP, Brazil) for 20 normal chewing strokes, as monitored by a single researcher. The chewed particles were then collected, dried and shaken at $2 \mathrm{~Hz}$ for $20 \mathrm{~min}$ in a sieving machine (Bertel, Caieiras, $\mathrm{SP}$, Brazil). While shaking, the particles passed through a 10-sieve stack with mesh sizes gradually decreasing from 5.6 to $0.5 \mathrm{~mm}$. The mass of the particles retained in each sieve was weighed on an analytical balance accurate to $0.001 \mathrm{~g}$ (Model 2060; Bel Engineering, Monza, Italy). The $X_{50}$ value for each subject was calculated by using the Rosin-Rammler equation (21). The $X_{50}$ value is a measure of MP and corresponds to the aperture of a theoretical sieve through which $50 \%$ of the weight of comminuted food could pass (21). Thus, the lower the $X_{50}$ value, the better the MP.

\section{$C A$}

Perceived $C A$ was measured by a subjective questionnaire evaluation to assess each subject's ability to chew seven types of food with different textures and consistencies, including bread, parmesan cheese, sausage, lettuce, peanuts, an apple, and raw carrots $(10,22)$. The questionnaire was based on a 100-mm VAS, with the ends of the scale representing "very easy" to "very difficult" to chew (0 and $100 \mathrm{~mm}$, respectively). Subjects were asked to think about their ability to chew these foods and draw a vertical mark on the horizontal VAS line at the point that best represented their ability. The distance $(\mathrm{mm})$ from the left end of the horizontal VAS line to the vertical mark made by the subject was measured (23) and used to calculate the CA mean values for all assessed groups. Lower scores represent greater CA (10).

The psychometric property of the VAS method is effective in measuring chewing ability, as well as general satisfaction, ability to speak and esthetics. This method has been used in subjects of all ages to assess their perceptions (23) with no need for special equipment and saving time.

\section{Statistical Analyses}

Continuous data were expressed as the mean \pm standard deviation (SD). The normality of each data distribution was assessed by skewness and kurtosis parameters and by Shapiro-Wilk tests. One-way ANOVA and Tukey-Kramer tests were used to analyze pain intensity, MBF, MP and CA. All statistical analyses were performed by the SAS software package (version 9.3, SAS Institute Inc., Cary, NC, USA). A p value $<0.05$ was considered statistically significant.

\section{Results}

Among the 48 selected individuals, most were patients seeking care for facial pain in the dental clinic of this Institution (41 subjects), while two were employees and five were students from the same Institution. The mean age and BMI of each experimental group are in Table 1. There was no statistical difference in age $(\mathrm{p}=0.3360)$ or $\mathrm{BMI}$ $(p=0.7538)$ among the craniofacial morphology groups. Table 2 shows the distribution of RDC/TMD diagnoses among test subjects. The diagnosis of articular disorders, such as disc displacement, arthralgia and osteoarthrosis, was accepted when at least one TMJ was affected. Interestingly, most patients fit more than one RDC/TMD diagnosis (Table 2). MBF values were the highest in the brachyfacial group, which differed significantly from the mesofacial and dolichofacial groups $(p=0.0001)$. No differences in MP or pain intensity values were observed (Table 3 ).

The craniofacial morphology groups did not differ in the CA of any of the food types, except for sausage $(p=0.0141$, Table 4). Brachyfacial patients reported the greatest ease to chew sausage, whereas dolichofacial patients reported the greatest difficulty to do so.

\section{Discussion}

The results of this study revealed that craniofacial morphology affects MBF but does not affect MP in females with painful TMDs. Among the craniofacial morphologies,

Table 1. Age and BMI of subjects in each craniofacial morphology group

\begin{tabular}{lcccc}
\hline & $\begin{array}{c}\text { Brachyfacial } \\
(\mathrm{n}=22)\end{array}$ & $\begin{array}{c}\text { Mesofacial } \\
(\mathrm{n}=13)\end{array}$ & $\begin{array}{c}\text { Dolichofacial } \\
(\mathrm{n}=13)\end{array}$ & $\begin{array}{c}\mathrm{p} \\
\text { value }\end{array}$ \\
\cline { 2 - 5 } Age (years) & $28 \pm 5.5$ & $26 \pm 5.6$ & $29 \pm 6.4$ & 0.3360 \\
BMI $\left(\mathrm{kg} / \mathrm{m}^{2}\right)$ & $24.04 \pm 4.04$ & $24.20 \pm 2.90$ & $24.93 \pm 3.78$ & 0.7538 \\
\hline
\end{tabular}

Data are shown as mean \pm standard deviation. One-way analysis of variance (ANOVA) significance threshold is $\mathrm{p}<0.05$. BMI $=$ Body mass index 
the self-perceived ability to chew was different only for one type of food (sausage), indicating that facial pattern possibly does not impair the ability to chew different types of food in painful TMD subjects.

The MBF values of the three craniofacial morphologies measured in this study were similar to those reported in previous studies, in which higher BF and muscular activity levels were observed during maximum clenching in subjects with shorter vertical craniofacial morphology (7). The higher reflex tonus in the masseter of short-face subjects (24) may explain why these individuals are able to bite with greater force, especially during jaw closure (24). However, our brachyfacial and mesofacial subjects with painful TMDs exhibited lower MBF values than those by pain-free subjects in a study by Custodio et al. (7). Recent studies $(25,26)$ have suggested that the presence of pain on the masseter muscle or TMJ has no effect on BF or mastication. Therefore, the disagreement between MBF values obtained in the present study and those found by Custodio et al. (7) may be independent of patient pain. Instead, this discrepancy may reflect sex differences, as half of the sample in Custodio et al. (7) was composed by male subjects. However, sex alone cannot explain this discrepancy because our MBF values were also lower than those reported in a study using subjects without TMD pain (8). The study by Gonçalves et al. (8), aimed at MBF in painfree females presenting disc displacement with reduction, showed higher values of MBF, indicating that pain may be a cause for the results of the present study.

Considering that pain solely cannot be responsible for reducing MBF (25), the inconsistencies between the present findings and those of Gonçalves et al. (8) maybe explained by the Integrated Pain Adaptation Model (IPAM) theory. This theory uses the interaction of biopsychosocial variables to explain motor control, such as BF (26). According to IPAM, when pain is present in the masticatory muscles, performing motor functions may differ between individuals depending on their psychological or emotional states (26). Thus, although the present study included no evaluation of

Data represent the mean \pm standard deviation. Different letters indicate significant differences among groups by ANOVA with Tukey-Kramer tests $(\mathrm{p}<0.05)$. 
the psychosocial parameters of subjects, our sample was different from that selected by Gonçalves et al. (8), who evaluated a convenience sample composed solely by female students and staff of Piracicaba Dental School. Since the sample of this study consisted mostly of patients who sought care for facial pain, it may be considered that these subjects presented different life stress factors and emotional states when compared to those from Gonçalves et al. (8), a condition that may explain the discrepancies in MBF values by IPAM.

Given the obtained MBF results of this study, it was anticipated that MP would also be affected by different craniofacial morphologies; however, this hypothesis was not confirmed. MP may be affected by the occlusal profile (5) and the number of occlusal contacts (1), which have been suggested as the major determinants of MP (4). Thus, given that all subjects of the present study were completely dentate with no malocclusion, the present result is not entirely surprising. Similarly to the present findings, others have also found no difference in MP values in subjects with symptomatic TMDs, strengthening the suggestion that the presence of pain does not reduce MP $(26,27)$.

The only significant difference in CA among the craniofacial morphology subjects was in their ability to chew sausage, which is considered softer than peanuts or raw carrots. This finding was unexpected because TMD patients, irrespective of their craniofacial morphology, usually complain of chewing difficulty (28), particularly with hard foods. A possible explanation may be related to the subjective nature of this CA test, wherein all seven foods were chosen from a list ranked in order of masticatory difficulty by full-denture wearers (10). Because our sample was composed of dentate subjects, they may have different dietary habits than denture wearers (29), which would influence the present results and considered a study limitation. However, the psychometric VAS method employed here was developed based on a study of chewing efficiency with dentate subjects $(23,30)$. It is commonly used in both experimental and clinical research, in conjunction with parametric statistical approaches (10). In addition, other factors, such as the type of food, which subjects may not be used to, could also affect the results. Overall, given the subjective nature of this test, the outcomes should be cautiously interpreted.

It is important to highlight that because the present study evaluated whether craniofacial morphology could affect mastication in painful TMD subjects, our experimental design did not include subjects wihtout TMDs. In the absence of a TMD diagnosis, dolichofacial patients still have reduced MP and BF values $(7,31)$. Although our data showed no difference in MP between subjects with different craniofacial morphologies, the $X_{50}$ values were higher than those obtained in a previous report (31), indicating poorer mastication by the our subjects, which may be explained by IPAM theory.

The psychosocial factors that affect mastication may go beyond the IPAM theory $(25,26)$ due to the multifactorial etiology of TMDs (32). Factors like anxiety and/or depression may affect masticatory muscle activity (33) and, therefore, chewing. However, the evaluation of psychosocial factors and psychological distress by RDC Axis II protocol was not used in this investigation, which could be considered a limitation. Although our outcomes were observed in a small number of participants, the significance detected in the obtained data confirms that this sample was sufficiently robust to support the findings. In conclusion, craniofacial morphology affects the MBF without impairing MP or CA in subjects with painful TMDs.

\section{Resumo}

A morfologia craniofacial afeta a performance mastigatória em individuos dentados saudáveis, mas pouco é conhecido sobre seus efeitos em pacientes com desordens temporomandibulares (DTMs) com sintomatologia dolorosa. Quarenta e oito pacientes do gênero feminino (idade média de $28 \pm 5,8$ anos) com DTM e sintomatologia dolorosa foram submetidas à radiografia cefalométrica lateral. Por meio da análise cefalométrica de Ricketts e método Vert, as voluntárias foram divididas segundo a morfologia crabiofacial em: braquifaciais $(n=22)$, mesofaciais $(n=13)$ ou dolicofaciais $(n=13)$. 0 Research Diagnostic Criteria for TMD (RDC/TMD) foi utilizado para diagnosticar a presença de DTM em cada paciente. A intensidade de dor foi reportada por cada paciente usando a escala visual analógica (EVA). A força máxima de mordida (FMM) foi medida com sensores de pressão colocados na região de primeiros molares. A performance mastigatória (PM) foi avaliada por meio da mastigação de material artificial confeccionado à base de silicone e determinada pelo tamanho da partícula, usando o método de fracionamento em peneiras. A habilidade mastigatória (HM) foi avaliada por meio de sete tipos de alimento e analisada por meio de EVA. Os dados foram analisados por ANOVA, seguido por teste de TukeyKramer $(p<0,05)$. A FMM foi diferente entre cada grupo, sendo que as pacientes braquifaciais apresentaram os maiores valores. Não houve diferença na PM entre os grupos. Os grupos somente diferiram quanto à HM para mastigar apenas um dos sete tipos de alimentos avaliados. Em resumo, a morfologia craniofacial afeta a FMM sem prejudicar a PM ou $\mathrm{HM}$ em pacientes com DTM e sintomatologia dolorosa.

\section{Acknowledgements}

This research was supported by São Paulo Research Foundation - FAPESP (Grant no. 2012/07282-9) and by the Fund to Support Teaching, Research, and Extension - FAEPEX/UNICAMP (Grant no. 519.292).

\section{References}

1. Abrahamsson C, Henrikson T, Bondemark L, Ekberg E. Masticatory function in patients with dentofacial deformities before and after orthognathic treatment - a prospective, longitudinal, and controlled study. Eur J Orthod 2015;37:67-72.

2. Bakke $M$, Handosttir R. Mandibular function in patients with temporomandibular joint pain: a 3-year follow-up. Oral Surg Oral Med Oral Pathol Oral Radiol Endod 2008;106:227-234.

3. N'gom PI, Woda A. Influence of impaired mastication on nutrition. J Prosthet Dent 2002;87:667-673.

4. Hatch JP, Shinkai RS, Sakai S, Rugh JD, Paunovich ED. Determinants of masticatory performance in dentate adults. Arch Oral Biol 2001;46:641-648. 
5. English JD, Buschang PH, Throckmorton GS. Does malocclusion affect masticatory performance? Angle Orthod 2002;72:21-27.

6. Sonnesen L, Bakke M, Solow B. Temporomandibular disorders in relation to craniofacial dimensions, head posture and bite force in children selected for orthodontic treatment. Eur J Orthod 2001;23:179-192.

7. Custodio W, Gomes SG, Faot F, Rodrigues Garcia RCM, Del Bel Cury AA. Occlusal force, electromyographic activity of masticatory muscles and mandibular flexure of subjects with different facial types. J Appl Oral Sci 2011;19:343-349.

8. Gonçalves TM, Sanchez-Ayala A, Ambrosano GM, Rodrigues Garcia $\mathrm{RCM}$. Female hormonal fluctuation and masticatory function in patients with disc displacement - a case-control study. Int J Prosthodont 2011;24:320-327.

9. van der Bilt A. Human oral function: a review. Braz J Oral Sci 2002;1:718.

10. Feine JS, Lund JP. Measuring chewing ability in randomized controlled trials with edentulous populations wearing implant prostheses. J Oral Rehabil 2006;33:301-308.

11. Ochiai KT, Hojo S, Nakamura C, Ikeda H, Garrett NR. Impact of facial form on the relationship between conventional or implant-assisted mandibular dentures and masticatory function. J Prosthet Dent 2011;105:256-265.

12. Kurita $H$, Ohtsuka $A$, Kurashina $K$, Kopp S. Chewing ability as a parameter for evaluating the disability of patients with temporomandibular disorders. J Oral Rehabil 2001;28:463-465.

13. Peck CC, Murray GM, Gerzina TM. How does pain affect jaw muscle activity? Integrated pain adaptation model. Aust Dent J 2008;53:201207.

14. Yamada K, Hanada K, Sultana MH, Kohno S, Yamada Y. The relationship between frontal facial morphology and occlusal force in orthodontic patients with temporomandibular disorder. J Oral Rehabil 2000;27:413421.

15. Sonnesen $L$, Bakke M, Solow B. Temporomandibular disorders in relation to cranifacial dimensions, head posture and bite force in childern selected for orthodontic treatment. Eur J Orthod 2001;23:179-192.

16. Pereira L, Gavião MB, Bonjardim LR, Castelo PM, van der Bilt A. Muscle thickness, bite force and craniofacial dimensions in adolescents with signs and symptoms of temporomandibular dysfunction. Eur J Orthod 2007;29:72-78.

17. Bósio JA, Burch JG, Tallents RH, Wade DB, Beck FM. Lateral cephalometric analysis of asymptomatic volunteers and symptomatic patients with and without bilateral temporomandibular joint disk displacement. Am J Orthod Dentofacial Orthop 1998;114:248-255.

18. Ricketts RM, Roth RH, Chaconas SJ, Schulhof RJ, Engel GA. Orthodontic diagnosis and planning. Denver: Rocky Mountain Data System; 1982.

19. Ricketts RM. Perspective in the clinical application of cephalometrics. The first fifty years. Angle Orthod 1981;51:115-150.

20. Fernandes $C P$, Glantz PO, Svensson SA, Bergmark A. A novel sensor for bite force determinations. Dent Mater 2003;19:118-126.
21. van der Bilt A, Olthoff LW, Bosman F, Oosterhaven SP. Chewing performance before and after rehabilitation of post-canine teeth in man. J Dent Res 1994;73:1677-1683.

22. Gonçalves TMSV, Campos CH, Rodrigues Garcia RCM. Mastication and jaw motion of partially edentulous patients are affected by different implant-based prostheses. J Oral Rehabil 2014;41:507-514.

23. De Grandmont $P$, Feine JS, Taché $R$, Boudrias $P$, Donohue $W B$, Tanguay $R$, et al.. Within-subject comparisons of implant-supported mandibular prostheses: Psychometric evaluation. J Dent Res 1994;73:1096-1104.

24. Naser Ud-Din S, Sowman PF, Sampson WJ, Dreyer CW, Turker KS. Masseter length determines muscle spindle reflex excitability during jaw-closing movements. Am J Orthod Dentofacial Orthop 2011;139:305-313.

25. Kumar A, Castrillon $E$, Svensson P. Can experimentally evoked pain in jaw or temporomandibular joint affect anterior bite force in humans? J Oral Facial Pain Headache 2015;29:31-40.

26. Shimada A, Baad-Hansen L, Svensson P. Effect of experimental jaw muscle pain on dynamic bite force during mastication. Arch Oral Biol 2015;60:256-66.

27. Vilanova LS, Gonçalves TM, Meirelles L, Rodrigues Garcia RCM. Hormonal fluctuations intensify temporomandibular disorder pain without impairing masticatory function. Int J Prosthodont 2015;28:7274.

28. Haketa T, Kino K, Sugisaki M, Amemori Y, Ishikawa T, Shibuya T, et al.. Difficulty of food intake in patients with temporomandibular disorders. Int J Prosthodont 2006;19:266-270.

29. Nowjack-Raymer RE, Sheiham A. Numbers of natural teeth, diet, and nutritional status in US adults. J Dent Res 2007;86:1171-1175.

30. Emmell S, Makawi K, Tanguay R, Feine JS, Lund JP. The influence of food type on experimental design in studies of chewing efficiency (abstract). J Dent Res 1991;70:277.

31. Farias Gomes SG, Custodio W, Faot F, Del Bel Cury AA, Rodrigues Garcia RCM. Masticatory features, EMG activity and muscle effort of subjects with different facial patterns. J Oral Rehabil 2010;37:813-819.

32. Manfredini D, Winocur E, Ahlberg J, Guarda-Nardini L, Lobbezoo F. Psychosocial impairment in temporomandibular disorders patients. RDC/TMD axis II findings from a multicenter study. J Dent 2010;38:765772.

33. Manfredini D, Fabbri A, Peretta R, Guarda-Nardini L, Lobbezoo F. Influence of psychological symptoms on home-recorded sleeptime masticatory muscle activity in healthy subjects. J Oral Rehabil 2011;38:902-911. 